'howler', as I discovered thanks to Dr. Jánossy's criticism. From elementary principles it must be $\frac{n-1}{2}+1=\frac{n+1}{2}$. In (4) you then get the factor $\frac{1}{2}+\frac{1}{2 n}+\frac{1}{n^{2}}$ in lieu of $1+\frac{1}{n^{2}}$.

Dublin Institute for Advanced Studies.

ERIVIN SCHRoedinger.

1 Jánossy, L., NATURE, 153, 165 (1944).

A TREATMENT of the higher order terms of the $n$-fold accidental rate is given by Prof. Schroedinger. A treatment leading to a slightly different result follows. Assume that pulses of the counters 1, 2, . . $n-I$ arrive in the time intervals $t_{1}, t_{1}+d t_{1}, \ldots$. $t_{n-1}, t_{n-1}+d t_{n-1}$ with

$$
0 \leqq t_{1} \leqq t_{2} \leqq \cdots \leqq t_{n-1} \leqq t,
$$

where $t$ is the resolving time. The above pulses together with one from counter $n$ at the time $t_{n}=t$ give rise to an $n$-fold coincidence. The probability per unit time of such an event is

$$
\boldsymbol{P}=N_{n}\left(N_{1} d t_{1}\right) \cdot\left(N_{2} d t_{2}\right) \ldots\left(N_{n-2} d t_{n-1}\right) . .
$$

To obtain a unique classification of the coincidences, we select all those coincidences under (1) for which the pulse of counter $I$ in the interval $t_{1}, t_{1}+d t_{1}$ is the first pulse of this counter to contribute to the coincidence. Both necessary and sufficient conditions for this are that no pulse of the counter 1 should arrive in the interval 0 to $t_{1}$. The probability for this is $\exp \left(-N_{1} t_{1}\right)$. Imposing similar conditions on the pulses coming from the other counters, one finds,

$P^{\prime}=\exp \left(-N_{1} t_{1}-N_{2} t_{2}-\ldots N_{n-1} t_{n-1}-N_{n} t\right) . P$, (2) where $P^{\prime}$ is the probability that the pulses from the counters arrive in specified time intervals and that the first pulses from the counters $1,2, \ldots n$ taking part in the coincidence arrive in a specified order. The total coincidence rate is obtained by integrating over the $t_{1}(i=1,2 \ldots n-1)$ and summing over the $n !$ permutations of the counters. We thus obtain

$$
R_{n}=N_{2} N_{2} \ldots N_{n} \Sigma e_{\text {perm. }}^{-N_{n} t} \int_{t_{1}=t_{2}=\ldots t_{n-1}=0}^{0 \leqq t_{1} \leqq t_{2} \ldots \leqq t} \ldots \int^{\infty} \ldots d t_{1} d t_{2} \ldots d t_{n-1}
$$$$
=\Sigma e^{-N_{n} t}\left(1-e^{-N_{1} t}\right) \ldots\left(1-e^{-N_{n_{-}} t}\right)
$$$$
\text { cyclic }
$$$$
\text { pyerm. }
$$

For $n=2$ one finds

$$
R_{2}=N_{1} e^{-N_{1} t}+N_{2} e^{-N_{2} t}-\left(N_{1}+N_{2}\right) e^{-\left(N_{1}+N_{2}\right) t} .
$$

Developing (3) into powers of $t$ one finds,

$$
\begin{aligned}
R_{n}=n . N_{1} & \ldots N_{n} t^{n-1} \\
& {\left[1-\left(\frac{1}{2}+\frac{1}{2 n}\right)\left(N_{1}+\ldots N_{n}\right) t\right]+\ldots }
\end{aligned}
$$

It is seen that (3) reduces to the expression I gave when neglecting higher powers of $t$. (The expression for $R_{n}$ giving the lowest power of $t$ only was also given by Eckart and Shonka ${ }^{2}$ some time ago.)

Regarding the practical applications, we note:

(1) In a counter arrangement consisting of $n$ counters, the accidental coincidences are usually due to various overlappings. One has to consider the overlap of a single pulse with a genuine $(n-1)$-fold coincidence ; further, one has to consider the overlap of two single counts with a genuine $(n-2)$-fold coincidence, etc. Thus one obtains for the rate of accidental coincidences an expression containing various powers of $t$. This expression may have no meaning if the higher powers in the terms due to low multiplicities are neglected.
(2) The expression (3) is only valid if the ineffective time following each pulse is short compared to $t$. In most practical cases, however, the ineffective time will be larger than $t$. In those cases the expression neglecting higher powers gives the correct rate.

Note added in proof.-The difference between the results as given above and as given by Prof. Schroedinger seems to be connected with the question as to what combination of pulses is recorded as one $n$-fold coincidence. My picture is as follows. Each pulse sets off the recorder for a time $t$. Any connected period of time for which the recorder is set off by all $n$ counters is counted as 'one coincidence'.

I am indebted to Prof. Schroedinger for an interesting correspondence.

Physical Laboratories,

University, Manchester.

${ }^{1}$ Jánossy, NATURE, 158, 165 (1944).

2 Eckart and Shonka, Phys. Rev., 53, 752 (1938).

\section{Donnan Membrane Potential}

IN a recent communication in Nature (July 17, 1943 , p. 76) I applied the Boltzmann distribution law to the Donnan membrane equilibrium between two compartments, compartment (1) containing colloidal (or non-diffusible) ions plus diffusible ions, compartment (2) containing only diffusible ions. From the equations obtained, it follows that at the same $p \mathrm{H}$ of the solution the ratio of the membrane potential $\left(E^{\prime}{ }_{m}\right)$ for a dibasic acid to the membrane potential $\left(E_{m}\right)$ for a monobasic acid should be

$$
\frac{E^{\prime}{ }_{m}}{\bar{E}_{m}}=\frac{\frac{R T}{N^{*} F} \cosh ^{-1} \frac{3 y+z}{3 x}}{\frac{B T}{N^{*} F} \cosh 1 \frac{2 y+z}{2 x}}=0.66 \text { nearly, }
$$

where $y$ is the concentration of positive, $y+z$ that of negative ions in compartment (1), $x$ the concentration of positive and negative ions in compartment (2), $N^{*}=\frac{1}{2}\left(N_{1}+N_{2}\right), N_{1}$ is the valency of the cation, $N_{2}$ is the valency of the anion, and $z$ is small. At the same $p H$ value of the solution, $z$ in the case of sulphuric acid is not equal to $z$ in the case of hydrochloric acid. According to Donnan, at the same $p \mathbf{H}$ of the protein solution, the value of $E^{\prime}{ }_{m} / E_{m}$ has been given as $\frac{2}{3}$, or 0.66 by Loeb. But this ratio should be 0.66 nearly, since the value of $z$ is different in the two cases.

University College of Science and Technology

$$
\text { S. G. Chaudhury. }
$$
92 Upper Circular Road,

$$
\text { Calcutta. Jan. } 29 .
$$

\section{Copper Carbonyl : a Correction}

RECENTIY ${ }^{1}$ we described a volatile compound, traces of which were formed when a variety of specimens of cuprous oxide were heated in carbon monoxide, as a carbonyl of copper. Further experiments have shown that the phenomena observed resulted from adventitious halide present in the oxides used.

P. L. RobINSON.

K. R. Stainthorpe.

King's College, University of Durham,

Newcastle upon Tyne. April 24.

1 Robinson and Stainthorpe, NatuRE, 153, 24 (1944). 